The Astrophysical Journal, 617:50-63, 2004 December 10

(C) 2004. The American Astronomical Society. All rights reserved. Printed in U.S.A.

\title{
PHOTOMETRIC PROPERTIES OF VOID GALAXIES IN THE SLOAN DIGITAL SKY SURVEY
}

\author{
Randall R. Rojas, ${ }^{1}$ Michael S. Vogeley, ${ }^{1}$ Fiona Hoyle, ${ }^{1}$ and Jon Brinkmann ${ }^{2}$ \\ Received 2004 July 2; accepted 2004 August 18
}

\begin{abstract}
Using a nearest neighbor analysis, we construct a sample of void galaxies from the Sloan Digital Sky Survey (SDSS) and compare the photometric properties of these galaxies to the population of nonvoid (wall) galaxies. We trace the density field of galaxies using a volume-limited sample with $z_{\max }=0.089$. Galaxies from the flux-limited SDSS with $z \leq z_{\max }$ and fewer than three volume-limited neighbors within $7 h^{-1} \mathrm{Mpc}$ are classified as void galaxies. This criterion implies a density contrast $\delta \rho / \rho<-0.6$ around void galaxies. From 155,000 galaxies, we obtain a subsample of 13,742 galaxies with $z \leq z_{\max }$, from which we identify 1010 galaxies as void galaxies. To identify an additional 194 faint void galaxies from the SDSS in the nearby universe, $r \lesssim 72 h^{-1}$ Mpc, we employ volume-limited samples extracted from the Updated Zwicky Catalog and the Southern Sky Redshift Survey with $z_{\max }=0.025$ to trace the galaxy distribution. Our void galaxies span a range of absolute magnitude from $M_{r}=$ -13.5 to -22.5 . Using SDSS photometry, we compare the colors, concentration indices, and Sersic indices of the void and wall samples. Void galaxies are significantly bluer than galaxies lying at higher density. The population of void galaxies with $M_{r} \lesssim M^{*}+1$ and brighter is on average bluer and more concentrated (later type) than galaxies outside of voids. The latter behavior is only partly explained by the paucity of luminous red galaxies in voids. These results generally agree with the predictions of semianalytic models for galaxy formation in cold dark matter models, which indicate that void galaxies should be relatively bluer, more disklike, and have higher specific star formation rates.
\end{abstract}

Subject headings: cosmology: observations — galaxies: photometry — galaxies: structure large-scale structure of universe - methods: statistical

\section{INTRODUCTION}

Since the discovery of the void in Bootes (Kirshner et al. 1981), with a diameter of $50 \mathrm{~h}^{-1} \mathrm{Mpc}$, and subsequent discoveries of voids in larger redshift surveys (Geller \& Huchra 1989; Pellegrini et al. 1989; da Costa et al. 1994; Shectman et al. 1996; El-Ad et al. 1996, 1997; Müller et al. 2000; Plionis \& Basilakos 2002; Hoyle \& Vogeley 2002), these structures have posed an observational and theoretical challenge. Because the characteristic scale of large voids was comparable to the depth of early redshift surveys, few independent structures were detected, making statistical analysis of their properties difficult. Likewise, the limitations of computing technology constrained early cosmological simulations to include only a few voids per simulation.

Whether voids are empty or not has been the question of recent debate. Peebles (2001) pointed out the apparent discrepancy between cold dark matter (CDM) models and observations. CDM models predict mass, and hence possibly galaxies inside the voids (Dekel \& Silk 1986; Hoffman et al. 1992). However, pointed observations toward void regions failed to detect a significant population of faint galaxies (Kuhn et al. 1997; Popescu et al. 1997; McLin et al. 2002). Surveys of dwarf galaxies indicate that they trace the same overall structures as larger galaxies (Bingelli 1989). Thuan et al. (1987), Babul \& Postman (1990), and Mo et al. (1994) showed that galaxies had common voids regardless of Hubble type.

Grogin \& Geller $(1999,2000)$ identified a sample of 149 galaxies that lie in voids traced by the Center for Astrophysics

\footnotetext{
1 Department of Physics, Drexel University, 3141 Chestnut Street, Philadelphia, PA 19104; rrojas@mercury.physics.drexel.edu, vogeley@drexel.edu, hoyle@venus.physics.drexel.edu.

2 Apache Point Observatory, P.O. Box 59, Sunspot, NM 88349-0059.
}

(CfA) survey. The void galaxies were found in the Century and $15 \mathrm{R}$ redshift samples. Grogin \& Geller showed that the void galaxies tended to be bluer and that a significant fraction of them were of late type. Their sample of 149 void galaxies covered a narrow range of absolute magnitude $(-20 \lesssim B \lesssim-17)$ of which 49 have a low-density contrast of $\delta \rho / \rho \leq-0.5$. Here we present a sample of $\sim 10^{3}$ void galaxies found in regions of density contrast $\delta \rho / \rho \leq-0.6$. This sample is large enough to allow comparison of void and wall galaxies with the same color, surface brightness profile, and luminosity to statistically quantify their differences. The range of absolute magnitude (Sloan Digital Sky Survey [SDSS] $r$ band) in our sample $\left(-22 \lesssim M_{r} \lesssim 13\right)$ is large enough to include faint dwarfs to giants.

In this paper, we introduce a new sample of void galaxies from the SDSS. The large sky coverage and depth of the SDSS provides us with the opportunity to identify for the first time more than $10^{3}$ void galaxies with $\delta \rho / \rho \leq-0.6$. In $\S 2$ we discuss the galaxy redshift samples that we use for this analysis. In $\S 3$ we describe our method for finding void galaxies. In $\S 4$ we present the results found from the comparison of the photometric properties of void and wall galaxies, and in $\S 5$ we interpret these results by comparing them to predictions from semianalytic modeling of structure formation and properties of different galaxy types. Finally, in $\S 6$ we present our conclusions.

\section{THE REDSHIFT SURVEYS}

The search for void galaxies requires a large threedimensional map of the galaxy density field. We extract a volume-limited sample from the SDSS data to map the galaxy density field and look for void galaxies in the full magnitudelimited sample. As the SDSS currently has a slicelike geometry, 
with each slice only $\sim 6^{\circ}$ thick, large voids of radius $\sim 10 h^{-1}$ Mpc $\left(h \equiv H_{0} / 100 \mathrm{~km} \mathrm{~s}^{-1} \mathrm{Mpc}^{-1}\right)$ can be detected only at comoving distances of $r \gtrsim 10^{2} h^{-1} \mathrm{Mpc}$ using the SDSS data alone. Therefore, to trace the local voids, we also extract a volume-limited sample from the combined Updated Zwicky Catalog (UZC; Falco et al. 1999) and Southern Sky Redshift Survey (SSRS2; da Costa et al. 1998). It should be noted that nearby void galaxies are not selected from the UZC and SSRS2 surveys. These surveys are only used to define the density field around SDSS galaxies that lie at distances $r \leq 72 h^{-1} \mathrm{Mpc}$.

To recap, we have two volume-limited samples, one from the SDSS and one from the combined UZC+SSRS2. These samples are used to define the galaxy density field only. Void galaxies are found from the magnitude-limited SDSS sample. We define the distant sample to be the SDSS magnitude-limited sample truncated at $100 h^{-1} \mathrm{Mpc} \leq r \leq 260 h^{-1} \mathrm{Mpc}$. The nearby sample is the SDSS magnitude-limited sample truncated at $r=72 h^{-1} \quad$ Mpc. Both magnitude-limited samples (nearby and distant) are constructed using the SDSS $r$ band. In this section we describe each of the surveys and samples in detail.

\subsection{The SDSS}

The SDSS is a wide-field photometric and spectroscopic survey. The completed survey will cover approximately $10^{4} \mathrm{deg}^{2}$. CCD imaging of $10^{8}$ galaxies in five colors and follow-up spectroscopy of $10^{6}$ galaxies with $r<17.77$ will be obtained. York et al. (2000) provides an overview of the SDSS, and Stoughton et al. (2002) describes the early data release (EDR) and details of the photometric and spectroscopic measurements made from the data. Abazajian et al. (2003) describes the first data release (DR1) of the SDSS. Technical articles providing details of the SDSS include descriptions of the photometric camera (Gunn et al. 1998), photometric analysis (Lupton et al. 1999), photometric system (Fukugita et al. 1996; Smith et al. 2002), photometric monitor (Hogg et al. 2001), astrometric calibration (Pier et al. 2003), selection of the galaxy spectroscopic samples (Strauss et al. 2002; Eisenstein et al. 2001), and spectroscopic tiling (Blanton et al. 2003a). A thorough analysis of possible systematic uncertainties in the galaxy samples is described in Scranton et al. (2002).

We examine a sample of 155,126 SDSS galaxies (Blanton et al. 2003b; SAMPLE10) that have both completed imaging and spectroscopy. The area observed by SAMPLE10 is approximately 1.5 times that of the DR1 (Abazajian et al. 2003). To a good approximation, the sample that we analyze consists of roughly three regions covering a total angular area of $1986 \mathrm{deg}^{2}$. Because of the complicated geometry of the SDSS sky coverage, the survey regions are best described in the SDSS coordinate system (see Stoughton et al. 2002). Where possible in this section we describe approximate limits in the more familiar equatorial coordinates. The first region is an equatorial stripe in the north Galactic cap (NGC). This stripe has a maximum extent of $7^{\circ} .5$ in the declination direction over the R.A. range $21^{\mathrm{h}} 30^{\mathrm{m}} \lesssim \alpha \lesssim 4^{\mathrm{h}} 10^{\mathrm{m}}$ and a maximum length of $120^{\circ}$ over the R.A. range $8^{\mathrm{h}} \lesssim \alpha \lesssim 16^{\mathrm{h}}$. The second region is in the south Galactic cap (SGC). There are three stripes, the boundaries of which are defined in the SDSS coordinate system. Each stripe is 2.5 wide in SDSS survey coordinates. One stripe is centered at $\delta=0^{\circ}$ and covers the R.A. range $20^{\mathrm{h}} 40^{\mathrm{m}} \lesssim \alpha \lesssim$ $2^{\mathrm{h}} 20^{\mathrm{m}}$. The other two stripes are above and below the equator and cover similar R.A. ranges. In survey coordinates these two stripes cover the range $-28^{\circ} \lesssim \lambda \lesssim 41^{\circ}, 130^{\circ} \lesssim \eta \lesssim 135^{\circ}$ and $-57^{\circ} \lesssim \lambda \lesssim 58^{\circ}, 155^{\circ} \lesssim \eta \lesssim 160^{\circ}$. The third large region is in the NGC. In the SDSS survey coordinates it covers the range $-48^{\circ} \lesssim \lambda \lesssim 50^{\circ}, 75^{\circ} \lesssim \eta \lesssim 85^{\circ}$. There are additional smaller stripes at $-37^{\circ} \lesssim \lambda \lesssim-22^{\circ}, 60^{\circ} \lesssim \eta \lesssim 70^{\circ}$ and $2^{\circ} \lesssim \lambda \lesssim$ $60^{\circ}, 90^{\circ} \lesssim \eta \lesssim 100^{\circ}$ (the boundary is an approximation because of the tiling geometry).

We correct the velocities of galaxies to the Local Group (LG) frame according to

$$
\Delta v=V_{\text {apex }}\left[\sin (b) \sin \left(b_{\text {apex }}\right)+\cos (b) \cos \left(b_{\text {apex }}\right) \cos \left(l-l_{\text {apex }}\right)\right],
$$

where $l_{\text {apex }}=93^{\circ}, \quad b_{\text {apex }}=-4^{\circ}$, and $V_{\text {apex }}=316 \mathrm{~km} \mathrm{~s}^{-1}$ (Karachentsev \& Makarov 1996). The magnitudes of the galaxies are $K$-corrected as described in Blanton et al. (2003c), and corrections for Galactic extinction are made using the Schlegel et al. (1998) dust maps. Finally, to convert redshifts into comoving distances we adopt an $\left(\Omega_{m}, \Omega_{\Lambda}\right)=(0.3,0.7)$ cosmology.

The decrease of observed galaxy density with distance in an apparent magnitude-limited galaxy sample might cause us to erroneously detect more voids at large distances. Therefore, we use a volume-limited subsample of the SDSS to define the density field of galaxies. This sample consists of galaxies with redshifts less than the redshift limit, $z_{\max }$, and SDSS $r$-band absolute magnitudes brighter than $M_{\text {crit }}$, where

$$
M_{\text {crit }}=r_{\text {lim }}-25-5 \log _{10}\left[d_{l}\left(z_{\max }\right)\right],
$$

$r_{\lim }{ }^{3}$ is the magnitude limit of the survey, and $d_{l}$ is the luminosity distance in units of $h^{-1} \mathrm{Mpc}$ at $z_{\max }$. We form a volume-limited sample of the SDSS with $z_{\max }=0.089$, with a corresponding absolute magnitude limit $M_{\text {crit }}=-19.87$ (in the SDSS $r$ band). The redshift limit $z_{\max }=0.089$ allows us to construct the largest possible volume-limited sample from the current SDSS sample. This volume-limited sample contains 22,866 galaxies, where the mean separation between these galaxies is $\sim 5.3 h^{-1} \mathrm{Mpc}$. For a $\left(\Omega_{m}=0.3, \Omega_{\Lambda}=0.7\right)$ cosmology, the redshift limit of $z_{\max }=$ 0.089 corresponds to a comoving distance of $260 h^{-1} \mathrm{Mpc}$. The lower bound of $100 h^{-1} \mathrm{Mpc}$ on the comoving distance is necessary because of the slicelike geometry of the early SDSS slices. Recall that voids of diameter $\gtrsim 10 h^{-1} \mathrm{Mpc}$ can only be found at $r \gtrsim 100 h^{-1} \mathrm{Mpc}$, as discussed in $\S 2$.

\subsection{The Updated Zwicky Catalog}

The UZC (Falco et al. 1999) includes a reanalysis of data taken from the Zwicky Catalog and CfA surveys (Zwicky et al. 1961, 1965; Zwicky \& Herzog 1962-1965; Zwicky \& Kowal 1968; Geller \& Huchra 1989; Huchra et al. 1990, 1995, 1999) together with new spectroscopic redshifts for some galaxies and coordinates from the digitized POSS II plates. Improvements over the previous catalogs include estimates of the accuracy of the CfA redshifts and uniformly accurate coordinates at the less than $2^{\prime \prime}$ level.

The UZC contains 19,369 galaxies. Of the objects with limiting apparent magnitude $m_{\mathrm{Zw}} \leq 15.5,96 \%$ have measured redshifts, giving a total number of 18,633 objects. The catalog covers two main survey regions: $8^{\mathrm{h}}<\alpha_{1950}<17^{\mathrm{h}}, 8^{\circ} .5<$ $\delta_{1950}<44^{\circ} .5$ in the NGC and $20^{\mathrm{h}}<\alpha_{1950}<4^{\mathrm{h}},-2.5<$ $\delta_{1950}<48^{\circ}$ in the SGC. We correct the velocities of the galaxies with respect to the $\mathrm{LG}$ as discussed in $\S 2.1$. The magnitudes of the galaxies are corrected for Galactic extinction using the Schlegel et al. (1998) dust maps, and the magnitudes are

\footnotetext{
${ }^{3}$ We use $r_{\text {lim }}=17.5$ instead of $r_{\text {lim }}=17.77$ in the construction of the volume-limited catalog to ensure that we have a uniform limit across all the data, since earlier stripes were only observed to $r_{\lim }=17.5$.
} 

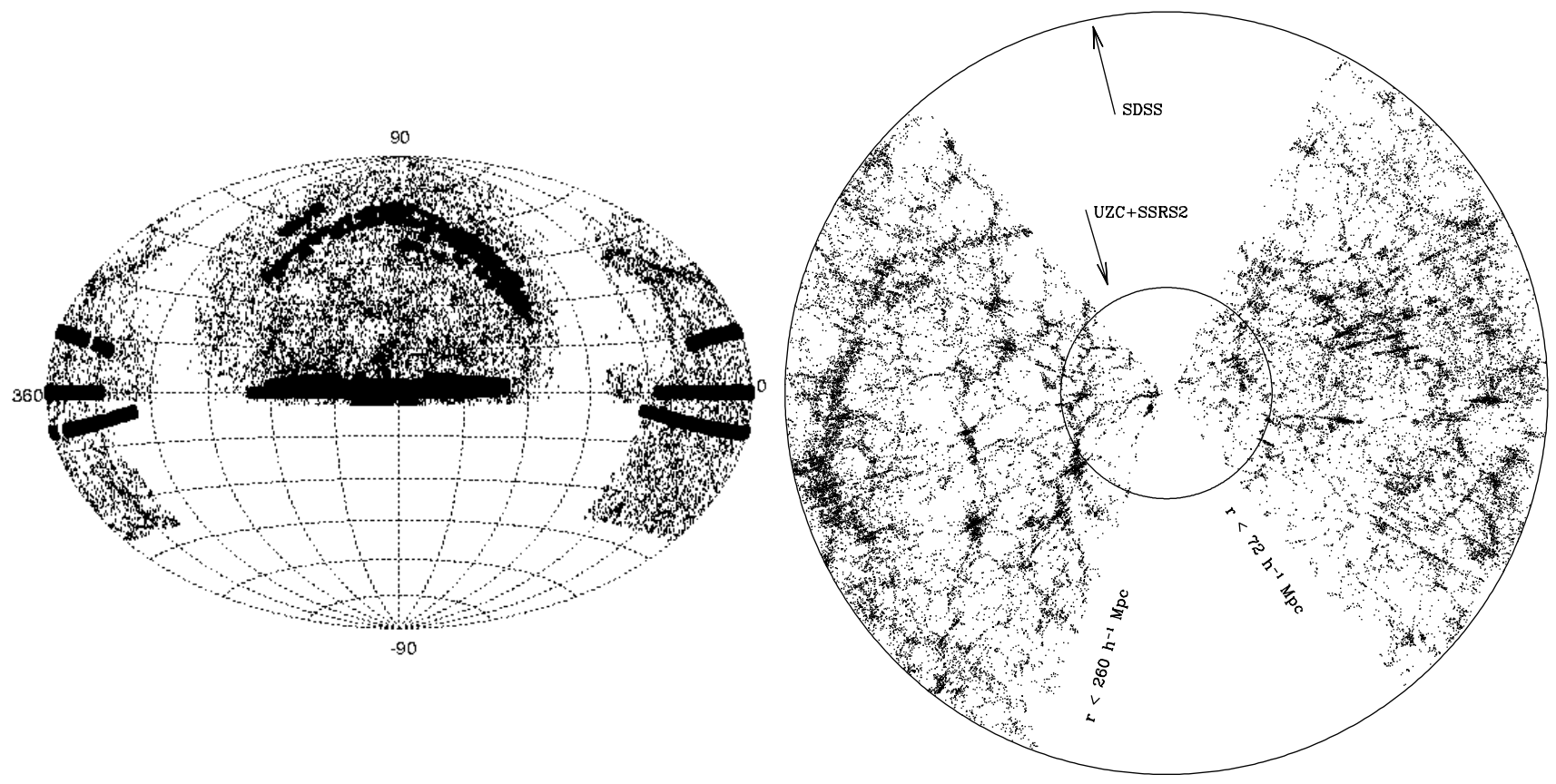

FIG. 1.-Left: Aitoff projection in celestial coordinates of the current SDSS galaxy redshift survey data (black sections) and the combined UZC and SSRS (dots). Approximate coordinates of each region are given in the text in $\S 2$. Right: Cone diagram of the flux-limited SDSS data with $|\delta| \lesssim 15^{\circ}$. The inner circle is drawn at $72 h^{-1} \mathrm{Mpc}$, which is the depth of the combined UZC and SSRS2 volume-limited sample. The outer circle is drawn at $260 h^{-1} \mathrm{Mpc}$, which is the depth of the SDSS volume-limited sample. In the region $100 h^{-1} \mathrm{Mpc}<r<260 h^{-1} \mathrm{Mpc}$ we use the SDSS data to trace the distribution of the voids. However, nearby the SDSS is currently limited in volume, as only narrow strips have been observed. Therefore, nearby we use the UZC+SSRS2 to trace the voids out to $72 h^{-1}$ Mpc.

$K$-corrected assuming $K=3$, which is appropriate for the $B$ filter and the median galaxy morphological type $\mathrm{Sab}$ ( Park et al. 1994; Pence 1976; Efstathiou et al. 1988).

We construct a volume-limited UZC sample with $z_{\max }=$ 0.025 , since this is the redshift at which the largest volumelimited sample can be obtained. This volume-limited sample contains 4924 galaxies and has a comoving depth of $\sim 76 h^{-1}$ $\mathrm{Mpc}$ and absolute magnitude limit of $M_{\mathrm{lim}} \leq-18.96\left(B_{\mathrm{Zw}}\right)$. To compare this limit to that of the SDSS, we translate a $B$-band magnitude into an approximate $r$-band magnitude of $M_{r}=$ -20.06 using $g-r=0.66$ and $g-B=-0.45$ from Fukugita et al. (1996). The absolute magnitude limit of the UZC sample is therefore slightly brighter than the SDSS limit. To ensure that this sample and the SSRS2 described below are equally deep, we cut back this sample to $72 h^{-1} \mathrm{Mpc}$.

\subsection{The SSRS2}

The SSRS2 galaxy sample (da Costa et al. 1998) was selected from the list of nonstellar objects in the Hubble Space Telescope Guide Star Catalog. The SSRS2 contains 3489 galaxies in the SGC over the angular region: $\delta_{1950} \leq-2.5$ and $b \leq-40^{\circ}$, covering a total of 1.13 sr with $m_{\mathrm{SSRS} 2} \leq 15.5$, where the zeropoint offset from the Zwicky magnitude system used in the UZC is approximately $m_{\mathrm{SSRS} 2}-m_{\mathrm{Zw}} \sim 0.10 \mathrm{mag}$ (Alonso et al. 1994).

We construct a volume-limited sample with the same redshift limit as for the UZC, $z_{\max }=0.025$ (same reason as discussed in $\S 2.2$ ) and (after adjustment of the zero point) $M \leq-18.96$. For our chosen cosmology, the depth of the sample is $\sim 73 h^{-1} \mathrm{Mpc}$, which we also cut back to $72 h^{-1} \mathrm{Mpc}$ as discussed in the case for the UZC sample. Therefore, both SSRS2 and UZC volumelimited samples have the same comoving depth.

As above ( $\S 2.1$ ), we correct galaxy velocities to the $\mathrm{LG}$ frame, apply the Galactic dust corrections based on the Schlegel et al. (1998) dust maps, and assume $K=3$ to $K$-correct the observed magnitudes. This volume-limited sample includes 725 galaxies.

The SSRS2 provides angular coverage in the SGC. The combined UZC+SSRS2 sample contains 5649 galaxies and sky coverage of $\sim 4.25 \mathrm{sr}$.

\subsection{Summary of Surveys}

Figure 1 (left) shows an Aitoff projection of the three surveys. The black sections show the SDSS galaxies, and the dots show the UZC+SSRS2 galaxies. This figure demonstrates that in terms of area, the SDSS is almost totally embedded in the $\mathrm{UZC}+\mathrm{SSRS} 2$ data apart from along the bottom edge of the northern equatorial slice and a small part of the southernmost slice. Therefore, the combined UZC+SSRS2 survey is useful for defining the large-scale galaxy density field around the SDSS sample out to a distance of approximately $72 h^{-1} \mathrm{Mpc}$.

Figure 1 (right) shows a cone diagram of the SDSS data with $|\delta| \lesssim 15^{\circ}$. The inner circle is drawn at $72 \mathrm{~h}^{-1} \mathrm{Mpc}$, which is the comoving depth of the combined UZC and SSRS2 volumelimited samples. The outer circle is drawn at $260 h^{-1} \mathrm{Mpc}$, which is the comoving depth of the SDSS volume-limited sample. Beyond $72 h^{-1} \mathrm{Mpc}$, the selection function (the number of observed galaxy density with distance) of these shallower surveys drops and the thickness (in the declination direction) of the SDSS itself is adequate to define the density field around the SDSS galaxies.

\section{IDENTIFYING VOID GALAXIES USING THE NEAREST NEIGHBOR STATISTICS}

We search for void galaxies in the SDSS using the nearest neighbor statistic. The two volume-limited samples (SDSS and UZC+SSRS2) are used to trace the voids. Any magnitudelimited galaxy that lies away from the boundary of the volumelimited sample and has less than three volume-limited sample 
neighbors in a sphere of $7 h^{-1} \mathrm{Mpc}$ is considered a void galaxy. We expand on each of these steps below.

\subsection{Proximity to Survey Boundary}

Galaxies in the magnitude-limited SDSS samples that lie near the boundaries of the volume-limited samples have systematically larger distances to their third nearest neighbors than galaxies that lie deep in the volume-limited samples. This is because potentially closer neighbors have not been observed/ included in the sample. These galaxies have a higher probability of being selected as void galaxies than the galaxies inside the survey. We correct for this bias in the following way. We generate a random catalog with the same angular and distance limits as the corresponding volume-limited sample (SDSS and UZC+ SSRS2) but with no clustering. We count how many random points lie around each of the magnitude-limited SDSS galaxies. If the density around a galaxy is less than a certain value, we reject it from the SDSS samples. This is explained further below.

We count how many random points $(N)$ lie in a sphere of size $r=3.5 h^{-1} \mathrm{Mpc}$ around each galaxy and compute the number density, $\rho=N / V$, where $V=\left[(4 \pi / 3) r^{3}\right]$. Since we know the number of random points, the solid angle, and the depth of the SDSS and UZC+SSRS2 surveys, we can compute the corresponding average density of random points, $\rho_{\text {random }}=$ $N /(\Omega / 3) r^{3}$. Galaxies with values of $\rho<\rho_{\text {random }}$ are rejected, as it is their proximity to the sample's boundaries that causes a low value of $\rho$.

We apply the above procedure twice: once when we compare the distant SDSS magnitude-limited sample with the SDSS random catalog and again when we compare the nearby SDSS magnitude-limited sample with the UZC+SSRS2 random catalog. The distant SDSS sample is reduced from 65,186 galaxies to 13,742 galaxies; the nearby SDSS sample is reduced from 3784 galaxies to 2450 galaxies. The nearby SDSS sample is cut less drastically, as the UZC+SSRS2 sample covers a greater area.

Because the SDSS is not finished, the angular selection function is complicated (see Fig. 1). An algorithm to quantify the fraction of galaxies that have been observed in any given region, i.e., the completeness, has been developed and is described in Tegmark et al. (2002). The completeness for any given $(\alpha, \delta)$ coordinate is returned, allowing a random cata$\log$ with the same angular selection function to be created. For the SDSS, the completeness within the regions that have been observed is typically greater than $90 \%$. The angular selection function for the combined UZC+SSRS2 sample is easier, as the surveys are finished and the completeness for the UZC is $\sim 96 \%$.

\subsection{Nearest Neighbor}

We classify galaxies that have a large distance to their $n$th nearest neighbor as void galaxies. We follow the work of ElAd \& Piran (1997) and Hoyle \& Vogeley (2002) and use $n=3$ rather than $n=1$ in the nearest neighbor analysis. Because galaxies are clustered, it is not unreasonable to expect that some galaxies in large-scale voids might be found in binaries or triplets. If we used $n=1$, then a pair of galaxies in an otherwise low-density environment would not be classified as void galaxies. Setting $n=3$ allows for a couple of bright neighbors but excludes galaxies in typical groups. Note that we do not make any corrections for peculiar velocities along the line of sight.
Therefore, we might underestimate the density of systems with large-velocity dispersions, which may pollute the void galaxy population. This effect could lead us to slightly underestimate the differences between the void and wall populations.

To identify void galaxies in the SDSS, we compute the distance from each galaxy in the apparent magnitude-limited sample to the third nearest neighbor in a volume-limited sample. In other words, the volume-limited sample is used to define the galaxy density field that traces voids and other structures. We compute the average distance to the third nearest neighbor, $\bar{d}_{\text {sep }}^{(3)}$, and the standard deviation, $\sigma_{\text {sep }}^{(3)}$, of this distance. We fix the critical distance $d_{\text {crit }}$ to be $7 h^{-1} \mathrm{Mpc}$, which is approximately equal to $\bar{d}_{\text {sep }}^{(3)}+1.5 \sigma_{\text {sep }}^{(3)}$ found from the two samples (the actual values are given in $\S \S 3.3$ and 3.4). This threshold is consistent with the criterion for defining wall and void galaxies in VOIDFINDER (Hoyle \& Vogeley 2002). Galaxies in the apparent magnitude-limited sample whose third nearest neighbor lies farther than $\geq d_{\text {crit }}=7 h^{-1} \mathrm{Mpc}$ are classified as void galaxies. We thereby divide the apparent magnitude-limited SDSS sample into two mutually exclusive subsamples, which we hereafter refer to as void and wall galaxies.

Note that the boundary of the void and wall samples is defined by throwing away galaxies that lie within $3.5 h^{-1} \mathrm{Mpc}$ of the survey edge, whereas galaxies are classified as void galaxies if they have less than three neighbors in a sphere of $7 h^{-1} \mathrm{Mpc}$. If we use $7 h^{-1} \mathrm{Mpc}$ to mark the boundary, then the volume available for finding void galaxies is decreased, especially at the near edge of the distant sample. We tolerate this inconsistency in order to have overlap between the near and distant samples in terms of the magnitude ranges that each sample probes. However, this means that there is a slightly higher probability of a galaxy being flagged as a void galaxy near the edges, rather than deep in the survey. To test what effect this has, we construct 10 mock volume- and flux-limited catalogs from the Virgo Consortium's Hubble Volume $z=0 \Lambda \mathrm{CDM}$ simulation (Frenk et al. 2000; Evrard et al. 2002) that have the same geometry as region 2. Following the procedure used in the survey data, we throw away galaxies in the flux-limited mock catalogs that lie within $3.5 h^{-1} \mathrm{Mpc}$ of the region's edge and then find the mock void galaxies. We then compute the average $N(r)$ distribution of the mock void and wall samples, where $N(r)$ is the normalized number of galaxies as a function of comoving distance, and show these in Figure 2 (left). It can be seen that within the errors the two distributions are similar. The exception is at the near edge where more mock galaxies are classified as void galaxies, as expected. Out to $125 h^{-1} \mathrm{Mpc}$ there are $50 \%$ more void galaxies than wall galaxies. This excess is only $4 \%$ of the whole void sample because most of the void galaxies are found at greater distances. The $N(r)$ plot for the data (Fig. 2, right) indicates a somewhat later ratio of void/wall galaxies at the near edge of the sample. From the test above, only $4 \%$ of the void galaxies might be erroneously flagged. The rest of the difference is due to largescale structure within the volume surveyed.

Thus we conclude that our procedure for identifying void galaxies and removing objects (both void and wall) near the survey boundaries does not produce any significant bias in the redshift distribution of void and wall galaxies. The difference is insufficient to generate the large observed differences between void and wall galaxies. In fact, dilution of the void galaxy sample can only decrease the apparent statistical significance of differences between the void and wall galaxy populations; i.e., the true differences between void and wall galaxies may be more severe than we find. The converse is not possible; this 

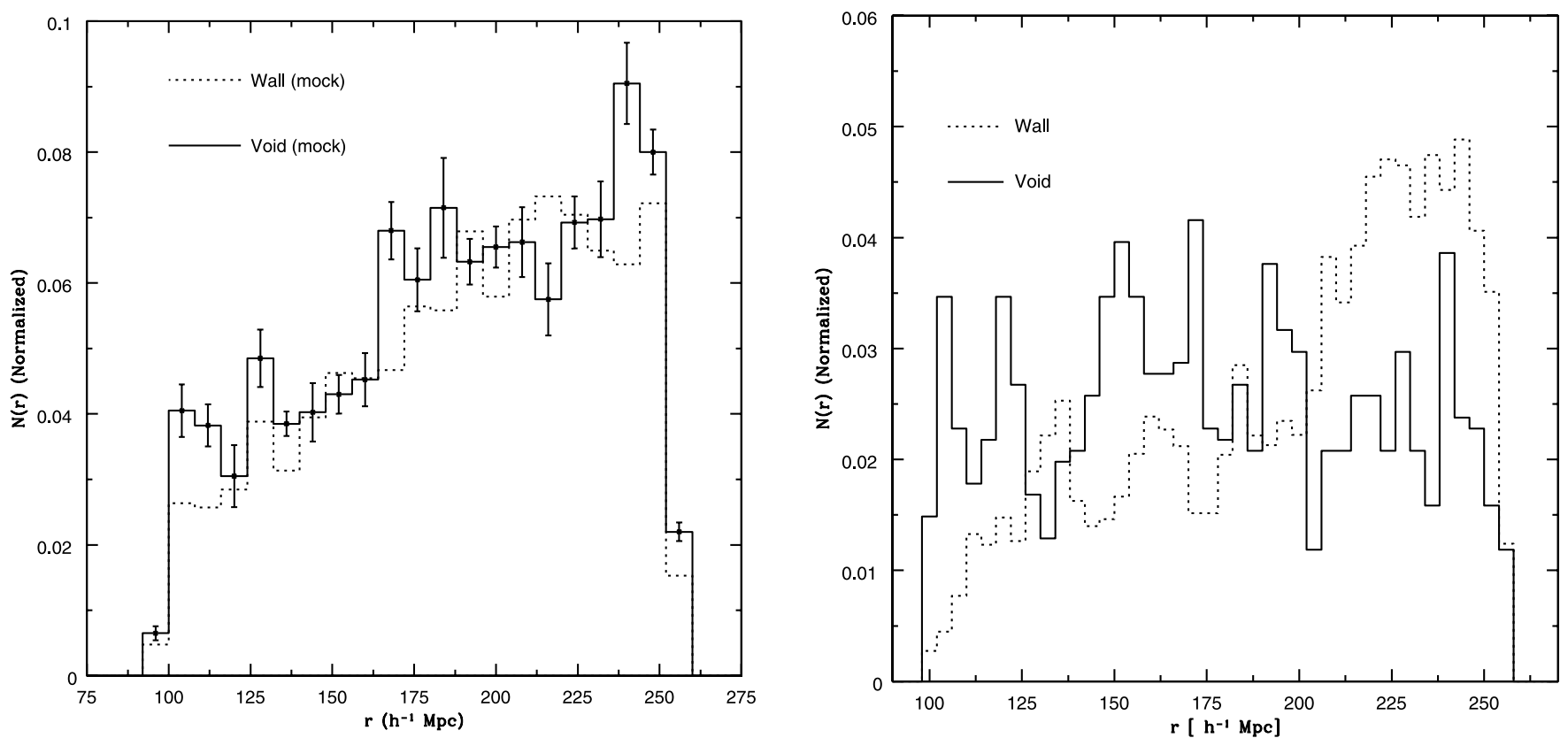

FIG. 2. $-N(r)$ distribution of void and wall galaxies from mock catalogs (left) and data (right). The left plot shows the $N(r)$ distribution as a function of comoving distance for the void (solid line) and wall (dotted line) mock samples. The mock samples are averaged over 10 independent realizations of region 2 (equatorial stripe in the NGC; see details in $\S 2.1$ ) from the Virgo Consortium's Hubble Volume $z=0 \Lambda$ CDM simulation (Frenk et al. 2000). The error bars are the $1 \sigma$ errors on the mock void galaxy samples. The right plot shows the same distribution for the distant void (solid line) and wall (dotted line) galaxy samples.

dilution could not cause the population differences that we observe. In $\S 5$ we discuss the impact of this dilution on our results. Also, tests with smaller "clean" samples show, as expected, a higher statistical significance, and results from the distant sample and the nearby sample, which suffers less from this effect because of a wider opening angle, show the same trends, which is further evidence that the dilution of the distant sample is a minor effect.

To test that our procedure identifies genuine void galaxies, we compute the mean, median, and upper bound of the density contrast $(\delta \rho / \rho)$ around void galaxies and compare these values to the emptiness of voids as defined by VOIDFINDER. The number of galaxies in the SDSS volume-limited sample is 22,866 , and the respective volume $V=(\Omega / 3)\left(r^{3}-r_{0}^{3}\right)=$ $(0.6 / 3)\left(260^{3}-100^{3}\right)=3.32 \times 10^{6} h^{-3} \mathrm{Mpc}^{3}$; therefore, the mean density is $\bar{\rho}=6.84 \times 10^{-3} h^{3} \mathrm{Mpc}^{-3}$. The void galaxies contain less than three neighbors in a sphere of $7 h^{-1} \mathrm{Mpc}$; thus, the density around the void galaxies is $\rho_{v} \leq 4 /(4 \pi / 3) 7^{3}=$ $2.78 \times 10^{-3} h^{3} \mathrm{Mpc}^{-3}$. Therefore, the density contrast around void galaxies in the distant sample is $\left(\rho_{v}-\bar{\rho}\right) / \bar{\rho} \leq-0.6$. This number is very similar for the nearby sample. It is an upper bound, as the median third nearest neighbor distance to the void galaxies is closer to $8 \mathrm{~h}^{-1} \mathrm{Mpc}$, giving values for the density contrast closer to $\delta \rho / \rho=-0.8$. This value is low, although not as low as that found by VOIDFINDER for the density contrast of the voids. Since we are centered on a galaxy and galaxies are clustered, we expect the density around void galaxies $\left(\rho_{\mathrm{vg}}\right)$ to be higher than the mean density of a void $\left(\bar{\rho}_{v}\right)$. Recall that the mean density of a void is about 0.1 times the mean density of the universe $\left(\bar{\rho}_{\text {universe }}\right)$, and since the correlation length $(\xi)$ on spheres of $8 h^{-1} \mathrm{Mpc}$ is $\sim 1\left(\sigma_{8} \sim 1\right)$, then $\rho_{\mathrm{vg}}\left(r \leq 7 h^{-1}\right.$ $\mathrm{Mpc})=\bar{\rho}_{v}\left[1+\xi\left(r=7 h^{-1} \mathrm{Mpc}\right)\right] \sim 2 \times \bar{\rho}_{v}$. In addition, void galaxies are typically found near the the edge of the void where the density is higher.

It is important to keep in mind that since most of the void galaxies will lie near the edges of voids, the typical density contrast around void galaxies is less extreme than the density contrast of the whole void region (see Fig. 11 in Benson et al. 2003). The average number of volume-limited galaxies in a sphere of $7 h^{-1} \mathrm{Mpc}$ around a wall galaxy is 25 compared to 2 around a void galaxy, demonstrating that void galaxies really are in highly underdense regions.

\subsection{Distant Void Galaxies}

For SDSS galaxies that lie in the distant SDSS sample, we use the SDSS volume-limited sample to define the galaxy density field. Using the third nearest neighbor $(n=3)$, we obtain $\left(\bar{d}_{\text {sep }}^{(3)}, \sigma_{\text {sep }}^{(3)}\right)=(3.6,2.10) h^{-1} \mathrm{Mpc}$, from which we obtain $d_{\text {crit }}=6.75 h^{-1} \mathrm{Mpc}$, which we round up to $7 h^{-1} \mathrm{Mpc}$. From the distant SDSS sample of 13,742 galaxies we find 1010 void galaxies. This sample of void galaxies is referred to as void galaxy distant (VGD). The sample of 12,732 nonvoid galaxies we label wall galaxy distant (WGD). The fraction of void galaxies in the distant sample is $\sim 8 \%$. This is only slightly higher than the fraction of void galaxies found by VOIDFINDER (Hoyle \& Vogeley 2002) and by El-Ad \& Piran (1997).

Figure 3 shows a redshift cone diagram of the SDSS wall galaxies (larger dots) and the corresponding void galaxies, VGD (small dots). We plot only galaxies with $|\delta| \lesssim 15^{\circ}$. Note that some of the void galaxies appear to be close to wall galaxies. This is merely a projection effect. All the void galaxies have less than three neighbors within a radius of $7 h^{-1} \mathrm{Mpc}$.

After obtaining the WGD and VGD samples, we split each void and corresponding wall galaxy sample into approximately equal halves by applying an absolute magnitude cut. In this case, the magnitude cut is done at $M_{r}=-19.5$, from which we obtained the corresponding subsamples, [WGD_b, VGD_b] $\left(M_{r} \leq-19.5, \mathrm{~b}=\right.$ bright $)$ and [WGD_f, VGD_f] $\left(M_{r}>-19.5\right.$, $\mathrm{f}=$ faint). The approximate range of absolute magnitudes covered by the subsamples is $-22 \lesssim M_{r} \leq-19.5$ for the bright half and $-19.5<M_{r} \leq-17.77$ for the faint half. Figure 4 shows the distribution of absolute magnitudes for the distant 


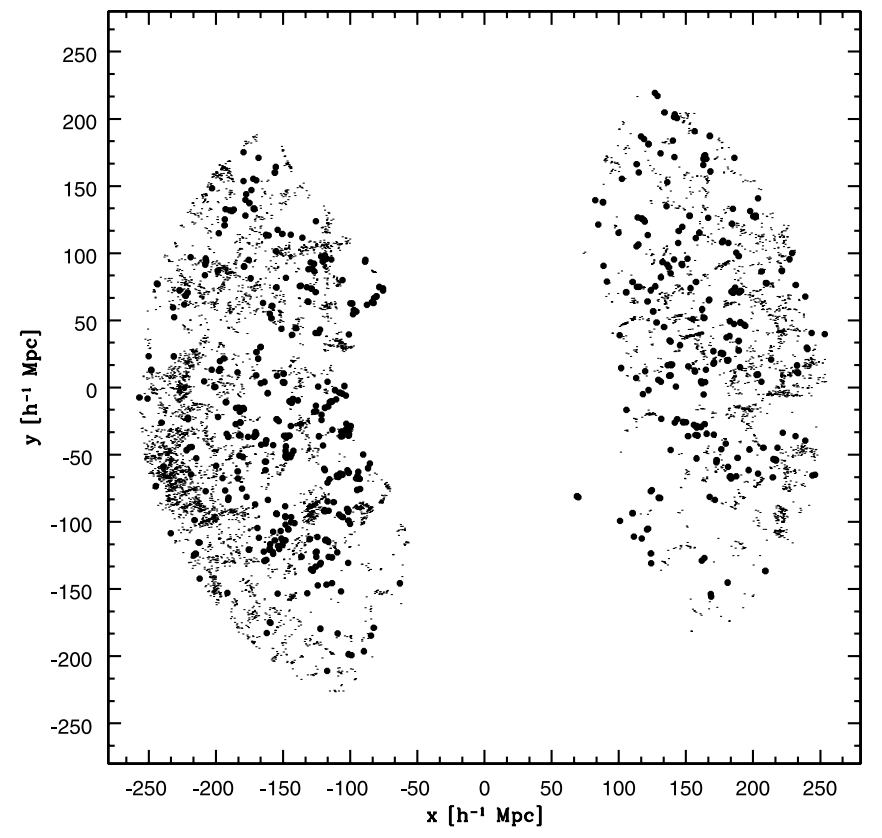

FIG. 3.-Redshift space distribution of void and wall galaxies. The larger dots show a cone diagram of the SDSS wall galaxies $\left(100 h^{-1} \mathrm{Mpc}<r<\right.$ $\left.260 h^{-1} \mathrm{Mpc}, M_{r} \lesssim-17.5\right)$. The smaller dots show the void galaxies from the apparent magnitude-limited sample $(r<17.5)$. We only plot galaxies with $|\delta| \lesssim 15^{\circ}$. Note that some of the black section appears to be close to magnitudelimited galaxies. This is, however, just a projection effect (we suppress the $z$-direction), and all void galaxies have less than three neighbors within a threedimensional radius of $7 \mathrm{~h}^{-1} \mathrm{Mpc}$.

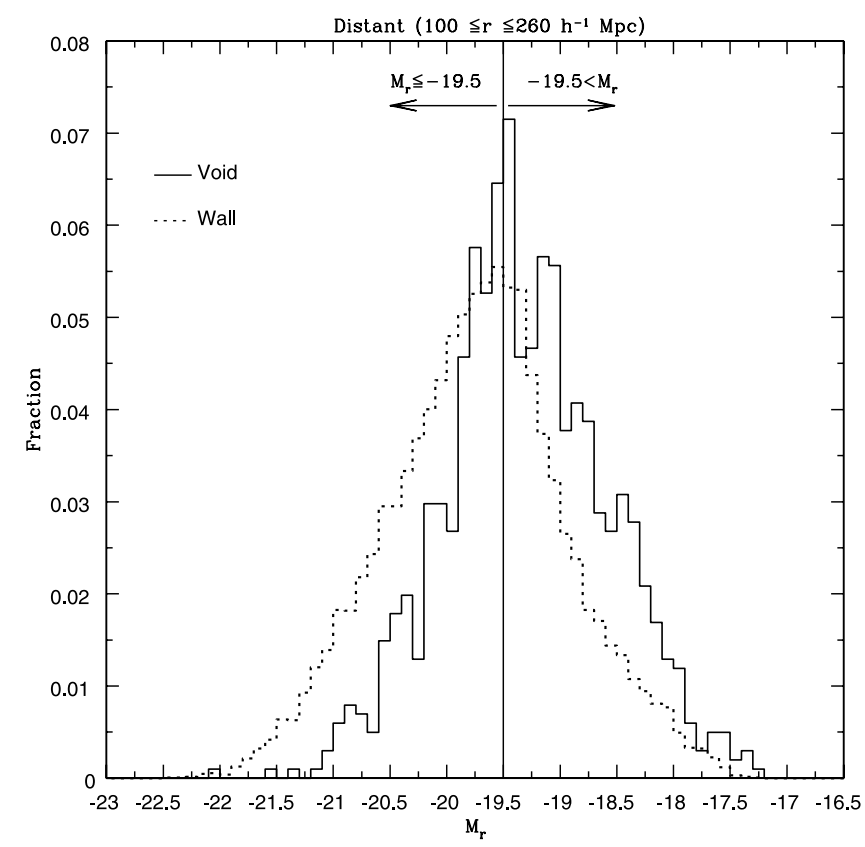

FIG. 4.-Distribution of the absolute magnitudes in the distant wall (WGD, dotted line) and void (VGD, solid line) galaxy samples. The parent sample is apparent magnitude-limited at $r \leq 17.5$ and redshift-limited at $z \leq 0.089$. We split both data sets at $M_{r}=-19.5$ to obtain the void galaxy subsamples [VGD_b $(b=$ bright $), V_{-}$VD_f $_{(}(\mathrm{f}=$ faint $)$ ] and wall galaxy subsamples [WGD_b, WGD_f]. The cut at $M_{r}=-19.5$ divides both the void and the wall galaxy samples into approximately equal halves. This histogram bins galaxies by $\Delta M=0.1$. The range of absolute magnitudes probed by the void galaxies that lie within this volume is $-22.0 \lesssim M_{r} \lesssim-17.2$.

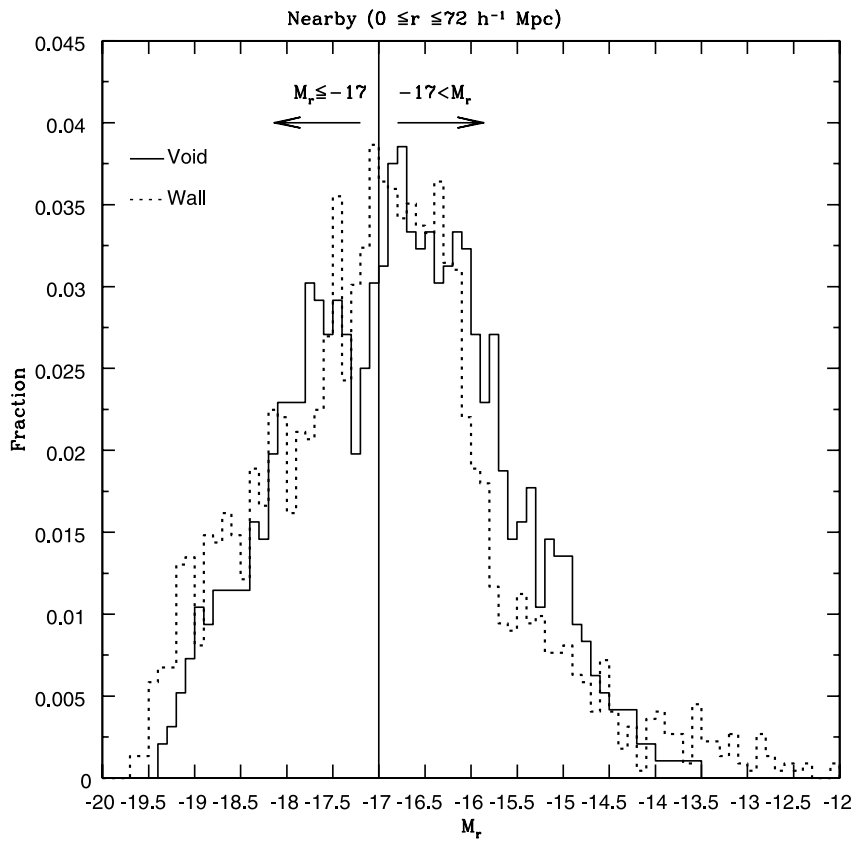

FIG. 5.-Distributions of absolute magnitudes in the nearby wall (WGN, dotted line) and void (VGN, solid line) galaxy samples. The parent sample is apparent magnitude-limited at $r \leq 17.5$ and redshift-limited at $z \leq 0.025$. Both data sets are split at $M_{r}=-17$ to obtain the void galaxy subsamples [VGN_b ( $\mathrm{b}=$ bright), VGN_f $(f=$ faint)] and wall galaxy subsamples [WGN_b, WGN_f]. The cut at $M_{r}=-17$ divides both the void and the wall samples into approximately equal halves. This histogram bins galaxies by $\Delta M=0.1$. The range of absolute magnitudes probed by the void galaxies that lie within this volume is $-19.7 \lesssim M_{r} \lesssim-13.0$.

samples. Note that the terms "bright" and "faint" in this context are used to describe the subsamples relative to their parent sample.

\subsection{Nearby Void Galaxies}

To find faint void galaxies, which are present in the SDSS sample only at small comoving distances, we use the $\mathrm{UZC}+\mathrm{SSRS} 2$ volume-limited sample to trace the voids, because the slicelike SDSS samples are too thin to detect threedimensional voids in this nearby volume.

The number of galaxies in the SDSS nearby sample, after applying the boundary corrections, is 2456 . We measure the distance to the third nearest UZC+SSRS2 volume-limited galaxy and obtain the values $\left(\bar{d}_{\text {sep }}^{(3)}, \sigma_{\text {sep }}^{(3)}\right)=(3.9,1.9) h^{-1} \mathrm{Mpc}$; hence the choice of $d_{\text {crit }}=7 h^{-1} \mathrm{Mpc}$ is still applicable. In this case we find 194 void galaxies. We refer to this void galaxy sample as void galaxy nearby (VGN) and the respective parent wall galaxy sample (after removing the respective void galaxies) as wall galaxy nearby (WGN).

We again apply an absolute magnitude cut to the VGN and WGN samples. For the nearby sample, this cut is done at $M_{r}=$ -17.0 (see Fig. 5). This cut divides the wall and respective void galaxy samples into approximately equal halves, which we label[WGN_b, VGN_b] $\left(M_{r} \leq-17.0, \mathrm{~b}=\right.$ bright $)$ and [WGN_f, VGN_f] $\left(M_{r}>-17.0, \mathrm{f}=\right.$ faint $)$. The range of absolute magnitudes included in each subsample is $-19.7 \lesssim M_{r} \leq-17.0$ for the bright half and $-17.0<M_{r} \lesssim-13.0$ (see Fig. 5) for the faint half. In this case the percent of void galaxies found is $8.6 \%$.

\section{PHOTOMETRIC PROPERTIES}

To examine whether void and wall galaxies have different photometric properties, we compare their colors $(u-r$ and 
TABLE 1

NeArby SAmple

\begin{tabular}{|c|c|c|c|}
\hline Property & $\begin{array}{l}\text { Void }^{\mathrm{a}} \\
\mu \pm \sigma_{\mu}\end{array}$ & $\begin{array}{c}\text { Wall (WGN) })^{\mathrm{b}} \\
\mu \pm \sigma_{\mu}\end{array}$ & K-S Probability $(P)$ \\
\hline \multicolumn{4}{|c|}{ Full $\left(-19.9 \leq M_{r} \leq-14.5\right),\left[N_{V}=194, N_{W}=2256\right]$} \\
\hline$g-r \ldots \ldots \ldots \ldots \ldots \ldots \ldots \ldots$ & $0.433 \pm 0.014$ & $0.490 \pm 0.004$ & 0.002 \\
\hline 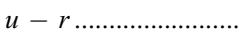 & $1.598 \pm 0.040$ & $1.764 \pm 0.013$ & 0.001 \\
\hline$r_{90} / r_{50} \ldots \ldots \ldots \ldots \ldots \ldots \ldots \ldots$ & $2.390 \pm 0.024$ & $2.390 \pm 0.007$ & 0.802 \\
\hline 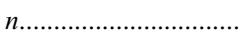 & $1.388 \pm 0.034$ & $1.456 \pm 0.004$ & 0.506 \\
\hline \multicolumn{4}{|c|}{ Bright $\left(M_{r} \leq-17.0\right),\left[N_{V}=76, N_{W}=1071\right]$} \\
\hline$g-r \ldots \ldots \ldots \ldots \ldots \ldots \ldots . .$. & $0.510 \pm 0.019$ & $0.549 \pm 0.006$ & 0.207 \\
\hline 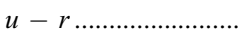 & $1.810 \pm 0.063$ & $1.930 \pm 0.019$ & 0.104 \\
\hline$r_{90} / r_{50} \ldots \ldots \ldots \ldots \ldots \ldots \ldots \ldots$ & $2.429 \pm 0.044$ & $2.421 \pm 0.011$ & 0.424 \\
\hline 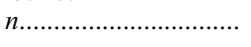 & $1.518 \pm 0.060$ & $1.626 \pm 0.004$ & 0.605 \\
\hline \multicolumn{4}{|c|}{ Faint $\left(M_{r}>-17.0\right),\left(N_{V}=118, N_{W}=1185\right)$} \\
\hline$g-r \ldots \ldots \ldots \ldots \ldots \ldots \ldots \ldots \ldots$ & $0.383 \pm 0.018$ & $0.436 \pm 0.006$ & 0.003 \\
\hline$u-r \ldots \ldots \ldots \ldots \ldots \ldots \ldots$ & $1.459 \pm 0.047$ & $1.611 \pm 0.016$ & 0.018 \\
\hline$r_{90} / r_{50} \ldots \ldots \ldots \ldots \ldots \ldots \ldots$ & $2.366 \pm 0.027$ & $2.361 \pm 0.008$ & 0.918 \\
\hline n & $1.305 \pm 0.039$ & $1.304 \pm 0.006$ & 0.509 \\
\hline
\end{tabular}

Notes.-Means, errors on the means, and K-S test probabilities that the void and wall galaxies are drawn from the same parent population for the photometric properties of void and wall galaxies in the nearby sample $\left(r<72 h^{-1} \mathrm{Mpc}\right)$. The number of galaxies (void and wall) in each sample and subsample are listed next to the magnitude range heading as $\left[N_{V}\right.$ (void), $N_{W}$ (wall)]. Small values of $P$ correspond to a low probability that the two samples are drawn from the same parent population. The K-S test shows that void galaxies appear to have different colors than wall galaxies. The void galaxies appear bluer than the respective wall galaxies in all cases, where the average difference between the means of the colors is about $2 \sigma_{\mu}$. However, the concentration and Sersic indices are not significantly different.

${ }^{a}$ VGN for full, VGN b for bright, VGN f for faint.

b WGN for full, WGN_

$g-r$ ), concentration indices, and Sersic indices. We compare the properties of wall and void galaxies in both the distant and the nearby samples. We also subdivide each sample by absolute magnitude and compare their properties further. The samples compared are therefore (1) distant: bright $\left(M_{r} \leq-19.5\right)$ [WGD_b, VGD_b], faint $\left(M_{r}>-19.5\right)$ [WGD_f, VGD_f], and full (undivided) void versus wall samples in each case, respectively, and (2) nearby: bright $\left(M_{r} \leq-17.0\right)$ [WGN_b, VGN_b], faint $\left(M_{r}>-17.0\right)$ [WGD_f, VGD_f], and full (undivided) void versus wall samples in each case, respectively.

We compute the means of the distributions and also the error on the mean to see if, on average, void and wall galaxies have the same colors, concentration indices, and Sersic indices. We also use the Kolmogorov-Smirnov (K-S) test to see if the void and wall galaxies could be drawn from the same parent population.

Tables 1 and 2 summarize the results of these tests for the nearby and distant samples, respectively. We present the results for the whole sample as well as for the samples split by absolute magnitude. The results are considered in detail below.

\subsection{Color}

The existence of strong correlations of galaxy type with density (Postman \& Geller 1984; Dressler 1980), galaxy type with color (Strateva et al. 2001; Baldry et al. 2004), and density with luminosity and color (Hogg et al. 2003; Blanton et al. 2003b) are well known; bright red galaxies tend to populate galaxy clusters and tend to be elliptical, while dim blue galaxies are less clustered and tend to be more disklike. This behavior is shown in an analysis of SDSS galaxy photometry by
Blanton et al. (2003b; see their Figs. 7 and 8), in which they find that the distribution of $(g-r)$ colors at redshift 0.1 is bimodal.

Of particular interest to us is the location of the void galaxies in color space. Because these galaxies evolve more slowly and interact less with neighboring galaxies than their wall galaxy counterparts, we might expect void galaxies to be dim, blue, and of low mass and to have high star formation rates (Benson et al. 2003). We consider two color indices, $u-r$ and $g-r$, because $g-r$ measures the slope of the spectrum and $u-r$ is sensitive to the UV flux and the $4000 \AA$ break. Since the $u$-band magnitudes can be noisy, by looking at $g-r$ and $u-r$ we are able to verify that the results are consistent and not affected by a low signal-to-noise ratio.

In Tables 1 and 2, we compare the photometric properties of the void galaxy samples to their respective wall galaxy samples. In Figures 6 and 7 we present normalized histograms of the color distributions. Solid lines correspond to the void galaxy samples, and the dotted lines represent the wall galaxies. In all cases (nearby, distant, and the bright and faint subsamples), we find that the void galaxy samples are on average bluer than the corresponding wall galaxy samples in both colors. If we look at the full samples, we find that the mean values of the two samples are significantly different. The nearby void galaxies have mean $u-r$ and $g-r$ colors that are at least $3 \sigma_{\mu}$ bluer than the wall galaxy samples. For the distant void galaxies, the differences in the means are about 4 times greater than for the nearby case.

When we split the nearby sample into the bright and faint samples, we see that it is at the faint end where there is the 
TABLE 2

Distant SAmple

\begin{tabular}{|c|c|c|c|}
\hline Property & $\begin{array}{l}\text { Void }^{\mathrm{a}} \\
\mu \pm \sigma_{\mu}\end{array}$ & $\begin{array}{l}\text { Wall }^{\mathrm{b}} \\
\mu \pm \sigma_{\mu}\end{array}$ & K-S Probability $(P)$ \\
\hline \multicolumn{4}{|c|}{ Full $\left(-22.5 \leq M_{r} \leq-17.77\right),\left[N_{V}=1010, N_{W}=12732\right]$} \\
\hline$g-r$ & $0.615 \pm 0.007$ & $0.719 \pm 0.002$ & $<10^{-4}$ \\
\hline 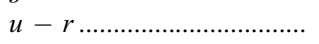 & $1.958 \pm 0.018$ & $2.219 \pm 0.005$ & $<10^{-4}$ \\
\hline 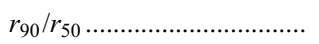 & $2.449 \pm 0.011$ & $2.571 \pm 0.004$ & $<10^{-4}$ \\
\hline$n$ & $1.718 \pm 0.024$ & $2.051 \pm 0.002$ & $<10^{-4}$ \\
\hline \multicolumn{4}{|c|}{ Bright $\left(M_{r} \leq-19.5\right),\left[N_{V}=409, N_{W}=7831\right]$} \\
\hline$g-r$ & $0.686 \pm 0.009$ & $0.765 \pm 0.002$ & $<10^{-4}$ \\
\hline$u-r$ & $2.126 \pm 0.026$ & $2.343 \pm 0.006$ & $<10^{-4}$ \\
\hline 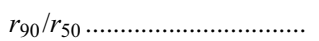 & $2.505 \pm 0.019$ & $2.656 \pm 0.004$ & $<10^{-4}$ \\
\hline$n$ & $1.908 \pm 0.042$ & $2.285 \pm 0.003$ & $<10^{-4}$ \\
\hline \multicolumn{4}{|c|}{ Faint $\left(M_{r}>-19.5\right),\left[N_{V}=601, N_{W}=4901\right]$} \\
\hline$g-r$ & $0.567 \pm 0.009$ & $0.645 \pm 0.003$ & $<10^{-4}$ \\
\hline$u-r$ & $1.844 \pm 0.024$ & $2.020 \pm 0.009$ & $<10^{-4}$ \\
\hline$r_{90} / r_{50} \ldots \ldots \ldots \ldots \ldots \ldots \ldots \ldots \ldots \ldots \ldots \ldots \ldots \ldots \ldots \ldots \ldots \ldots$ & $2.411 \pm 0.013$ & $2.435 \pm 0.005$ & 0.211 \\
\hline$n$ & $1.589 \pm 0.028$ & $1.677 \pm 0.003$ & $<10^{-4}$ \\
\hline
\end{tabular}

Notes.-Means, errors on the means, and K-S test probabilities that the void and wall galaxies are drawn from the same parent population for the photometric properties of void and wall galaxies in the distant sample $\left(100 h^{-1} \mathrm{Mpc} \leq r \leq 260 h^{-1} \mathrm{Mpc}\right)$. The number of galaxies (void and wall) in each sample and subsample are listed next to the magnitude range heading as $\left[N_{V}\right.$ (void), $N_{W}$ (wall)]. Small values of $P$ correspond to a low probability that the two samples are drawn from the same parent population. In this case, the K-S test shows that the void and wall galaxies are drawn from different populations based on both color and morphology (CI and SBP). The differences between the means of the different parameters measured are on average greater than $5 \sigma_{\mu}$, except for the $\mathrm{CI}$ in the faint subsample, where the difference is $\sim 2 \sigma_{\mu}$. Void galaxies are on average bluer and more disklike than wall galaxies.

${ }^{a}$ VGD for full, VGD_b for bright, VGD_f for faint

${ }^{b}$ WGD for full, WGD_b for bright, WGD_f for faint.

greatest difference between void and wall galaxies. The significance of the K-S test is reduced because of the smaller number of galaxies in each sample. The nearby bright and faint void galaxies are at least $2 \sigma_{\mu}$ bluer than the wall galaxies. The differences between the nearby void and wall galaxies are not as pronounced as in the distant samples because we are shot-noise-limited by how many clusters there are in the small nearby volume. In the distant sample it is very unlikely that the wall and void galaxies in both the bright and the faint subsamples are drawn from the same parent population $\left(P<10^{-4}\right)$.

We assess the statistical significance of differences in the color distributions using a K-S test (the values of $P$, the probability that the two samples are drawn from the same parent population, are given in the last column of Tables 1 and 2). The probability that the void and wall galaxies are drawn from the same parent population is low: $P<0.002$ in the nearby case and $P<10^{-4}$ in the distant case. In the distant sample it is very unlikely that the wall and void galaxies in both the bright and the faint subsamples are drawn from the same parent population $\left(P<10^{-4}\right)$.

\subsection{Concentration Index}

To compare morphological properties of void and wall galaxies, we examine the distribution of concentration indices measured by the SDSS photometric pipeline (Lupton et al. 1999, 2001; Stoughton et al. 2002; Pier et al. 2003). The concentration index (CI) is defined by the ratio $\mathrm{CI} \equiv r_{90} / r_{50}$, where $r_{90}$ and $r_{50}$ correspond to the radii at which the integrated fluxes are equal to $90 \%$ and $50 \%$ of the Petrosian flux, respectively. A large value of CI corresponds to a relatively diffuse galaxy and a small value of $\mathrm{CI}$ to a highly concentrated galaxy. The CI has been shown to correlate well with galaxy type (Strateva et al. 2001; Shimasaku et al. 2001). Spiral galaxies are usually found to have small CIs $(\leqslant 2.5)$, whereas elliptical galaxies have larger CIs $(\gtrsim 2.5)$. This bimodal behavior of the CI can be clearly seen in Strateva et al. (2001; see Fig. 8).

Figure 8 shows histograms of CI for void and wall galaxies for both the nearby and the distant samples along with the respective bright and faint subsamples. Tables 1 and 2 show the mean, error on the mean, and the K-S statistic found when comparing the wall and void galaxies.

In the nearby samples, the void and wall galaxies are not distinguished by this morphological parameter. In Table 1, we find that the mean values of CI are very similar. The probability that the distributions of CIs of void and wall subsamples are drawn from the same parent population approaches unity and 0.5 for the faint and bright subsamples, respectively. The top row of plots in Figure 8 shows that there is indeed little difference between the distributions of CI for the void and wall galaxies in these samples.

We find that void galaxies have, on average, significantly smaller CIs in the bright half of the distant samples. There are more wall than void galaxies at large values of $\mathrm{CI}(\mathrm{CI} \gtrsim 2.5)$. The means differ by more than $7 \sigma_{\mu}$. In Figure 8 , in the bottom row, all three dotted curves show this behavior. In Table 2, it is clear that in the full sample and the bright sample, the wall and void galaxies have significantly different CI distributions. A 

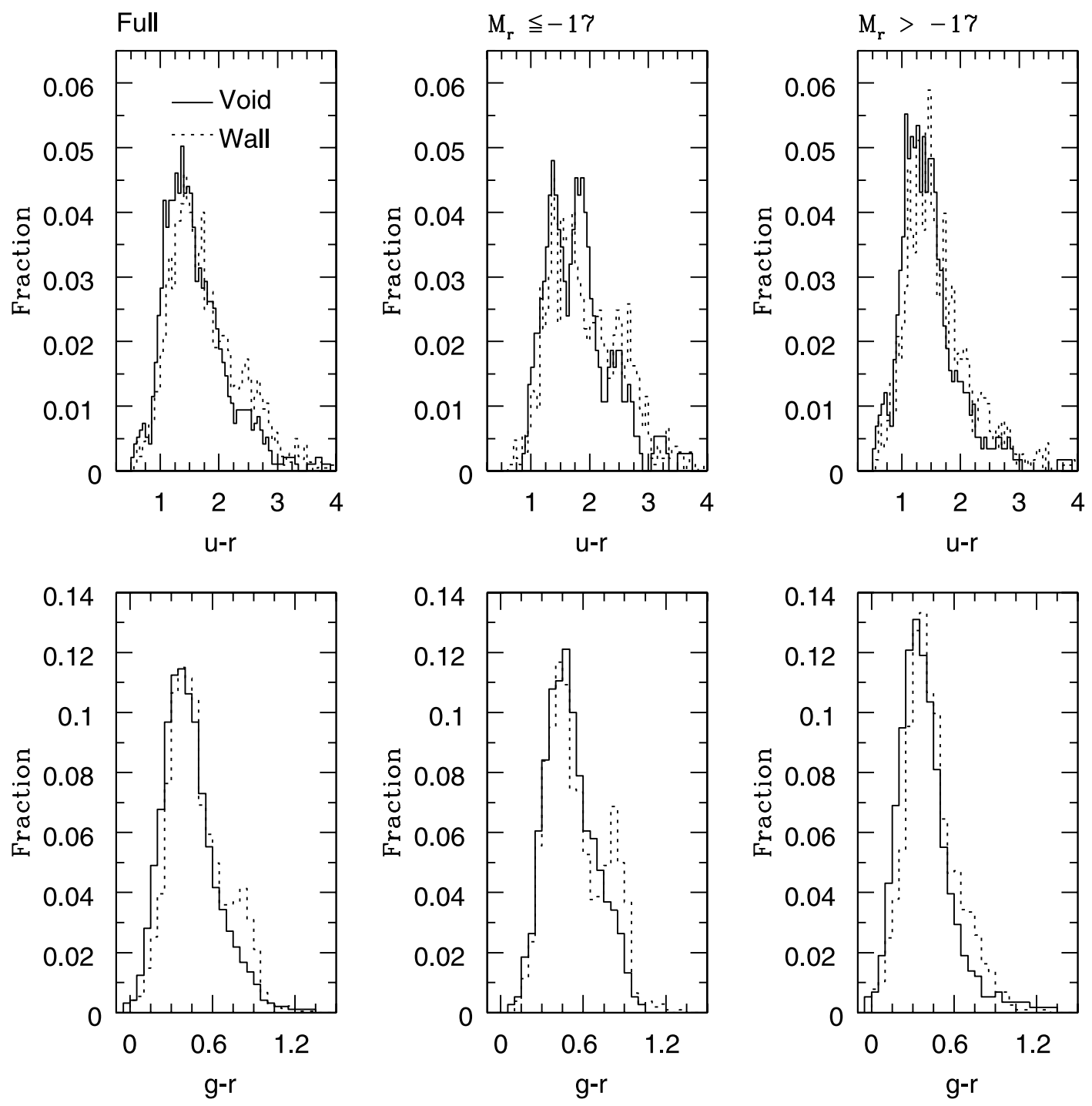

FIG. 6. - Color distributions of nearby void galaxies (solid lines) compared to the nearby wall galaxies (dotted lines) in two optical colors, $u-r$ (top row) and $g-r$ (bottom row). The first, second, and third columns are the undivided (full), bright $\left(M_{r} \leq-17\right)$, and faint $\left(-17<M_{r}\right.$ ) samples, respectively. The fraction of galaxies per 0.05 bin of color is shown on the $Y$-axis. In all cases, the solid curves are shifted to the left; i.e., on average, the void galaxies are bluer than wall galaxies (see Table 1 ). A $\mathrm{K}-\mathrm{S}$ test reveals that in the case of the faint subsample and full sample it is very unlikely $(\bar{P} \lesssim 0.007)$ that the full void and wall galaxy samples are drawn from the same respective parent populations. In the bright subsample, we see an excess of luminous red galaxies at $g-r \sim 0.9$ that is not present in the void galaxy histogram.

K-S analysis of the bright subsample reveals that there is a probability of less than $10^{-4}$ that the void and wall galaxies are drawn from the same parent population. In the case of the distant faint void and wall galaxy samples the results are consistent.

\subsection{Sersic Index}

As another measure of morphology of void and wall galaxies, we examine the Sersic index (Sersic 1968), found by fitting the functional form $I(r)=I_{0} \exp \left(-r^{1 / n}\right)$, where $n$ is the Sersic index itself, to each galaxy surface brightness profile (SBP). With this form, $n=1$ corresponds to a purely exponential profile, while $n=4$ is a de Vaucouleurs profile. We use the Sersic indices as measured by Blanton et al. (2003b) for the SDSS galaxies.

In Figure 9, we plot histograms of Sersic indices measured for all the samples. Statistics of these distributions and the results of comparison of void and wall subsamples are listed in Tables 1 and 2 . In the nearby survey volume, we find $\bar{n} \lesssim 1.5$ for all void and wall galaxy subsamples, and there are no statistically significant differences between the distributions. The top panels of Figure 9 show histograms of the Sersic index; the distributions of the void (solid lines) and wall (dotted line) galaxies appear very similar.

We find significant differences between void and wall galaxies in the distant samples. The bottom panels in Figure 9 show the distribution of Sersic indices for the void and wall galaxies. For the more distant void galaxies, we find in Table 2 that $\bar{n}_{\text {distant }}=1.7$, which is higher than what was found for the nearby void galaxies $\left(\bar{n}_{\text {nearby }}=1.4\right)$. A K-S test reveals that the void galaxies are distinct from the wall galaxies. There is a probability of $P<10^{-4}$ in the fainter $\left(M_{r}>-19.5\right)$, brighter $\left(M_{r}<-20.3\right)$, and full samples that the void and wall galaxies are drawn from the same parent population. The means of the Sersic indices of the void and wall galaxies differ by at least $3 \sigma_{\mu}$.

\section{DISCUSSION}

The above analysis clearly shows that there is a difference in the photometric properties of void and wall galaxies. Void galaxies are fainter and bluer than wall galaxies in all cases. 

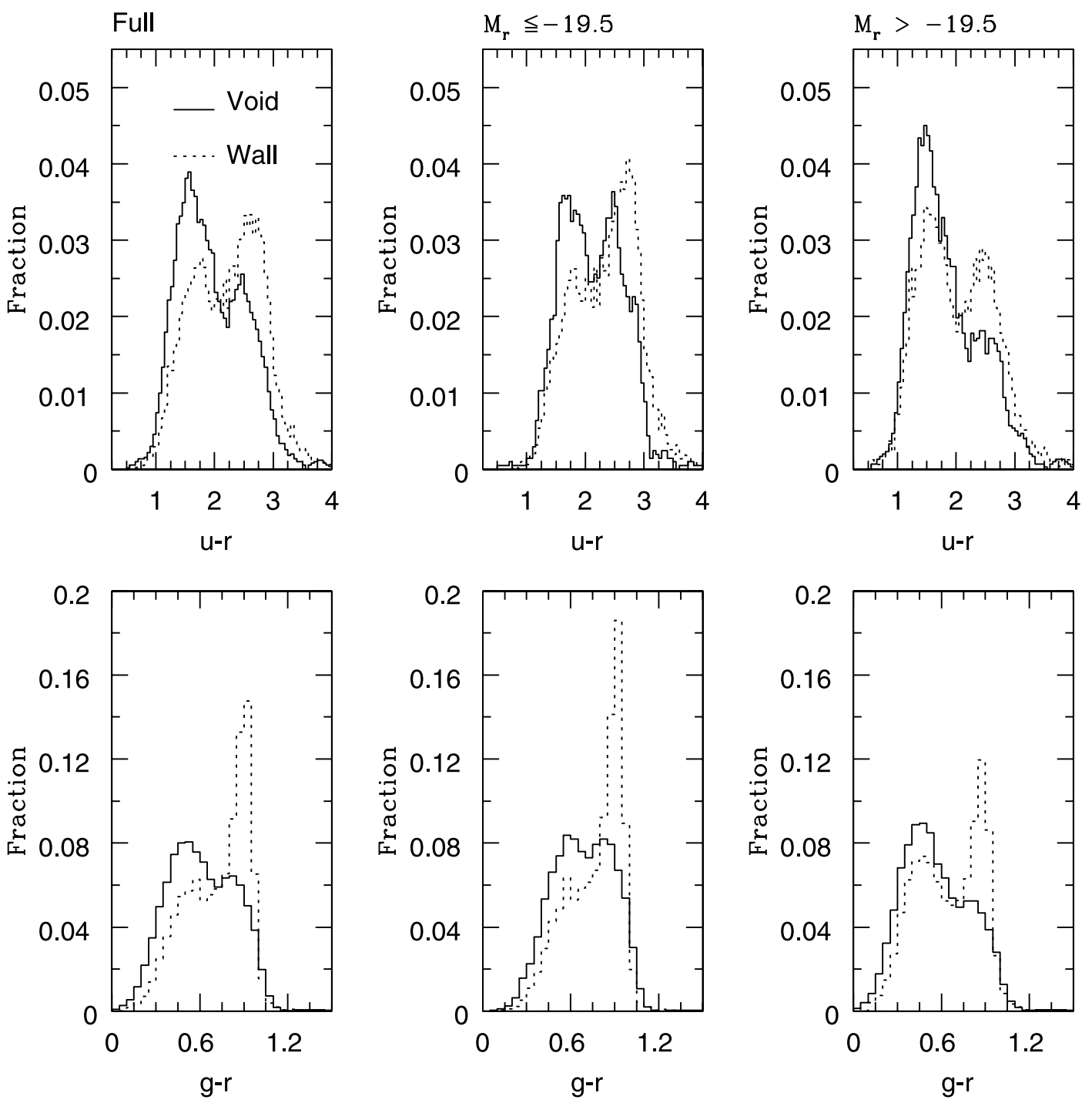

FIg. 7.-Color distributions of distant void galaxies (solid lines) compared to the distant wall galaxies (dotted lines) in two optical colors, $u-r$ (top row) and $g-r$ (bottom row). The left, middle, and right panels are the undivided (full), bright $\left(M_{r} \leq-19.5\right)$, and faint $\left(-19.5<M_{r}\right)$ samples, respectively. The fraction of galaxies per 0.05 bin of color is shown on the $Y$-axis. In all cases, the solid curves are shifted to the left; i.e., on average, the void galaxies are bluer than wall galaxies (see Table 2 ). A K-S test reveals that it is very unlikely $\left(P<10^{-4}\right)$ that the void galaxy and wall galaxy samples are drawn from the same parent populations. We clearly see an excess of luminous red galaxies in the wall galaxy histograms as a peak at $g-r \sim 1.0$.

Previous observational studies (e.g., Vennik et al. 1996; Pustilnik et al. 2002; Popescu et al. 1997) suggested that isolated galaxies in voids can be distinguished from nonvoid galaxies based on their color with a large enough sample. Here we provide such a sample and even extend the analysis to compare subsamples of void and nonvoid galaxies of similar luminosity and SBP.

Nearby, the question might be raised as to whether it is the faintest void galaxies that are particularly blue and whether these galaxies dominate the statistics. To test for this, the nearby sample is cut at -17.0 , reducing the range of absolute magnitude in each bin, and again the void galaxies are bluer than the wall galaxies. A further test was made in which the galaxies were divided into bins of $\delta M=1 \mathrm{mag}$, and still the void galaxies are bluer in every bin; thus the differences in color are not dominated by the tail of the distribution. Void galaxies are genuinely bluer than wall galaxies of the same luminosity.

In the distant sample the differences in color are only partly explained by the paucity of luminous red galaxies in voids. The average galaxy in the distant sample has an absolute magnitude of around -19.5 , which is more than a magnitude fainter than an $M^{*}$ galaxy in the $r$ band $\left(M^{*}=-20.80\right.$; Blanton et al. 2001). Galaxies that are thought of as bright red cluster elliptical galaxies are typically brighter than $M^{*}$. In the full sample, the faint sample, and the bright sample (and in the $\delta M=1 \mathrm{mag}$ test), void galaxies are still bluer than wall galaxies.

In $\S 3.2$ we noted a small excess of void galaxies near the inner boundary of the volume that encloses the distant samples. We predicted that this might affect the purity of our void galaxy samples and thereby lower the apparent statistical significance of differences between the void and wall galaxy populations. To test for this effect, we redo selected analyses to compare the photometric properties of void and wall galaxies in the range of comoving coordinate distance from $r=160$ to $260 h^{-1} \mathrm{Mpc}$, far from the region where the excess is observed near the $r=100 h^{-1} \mathrm{Mpc}$ inner boundary. We find that the differences between the photometric properties of void and wall galaxies are indeed larger for galaxies in this more restricted redshift range. For example, in $u-r, u-g$, and $g-r$ the differences rise to greater than $7 \sigma_{\mu}$. The sense of these differences is the same as for the larger sample; void galaxies are bluer and of 

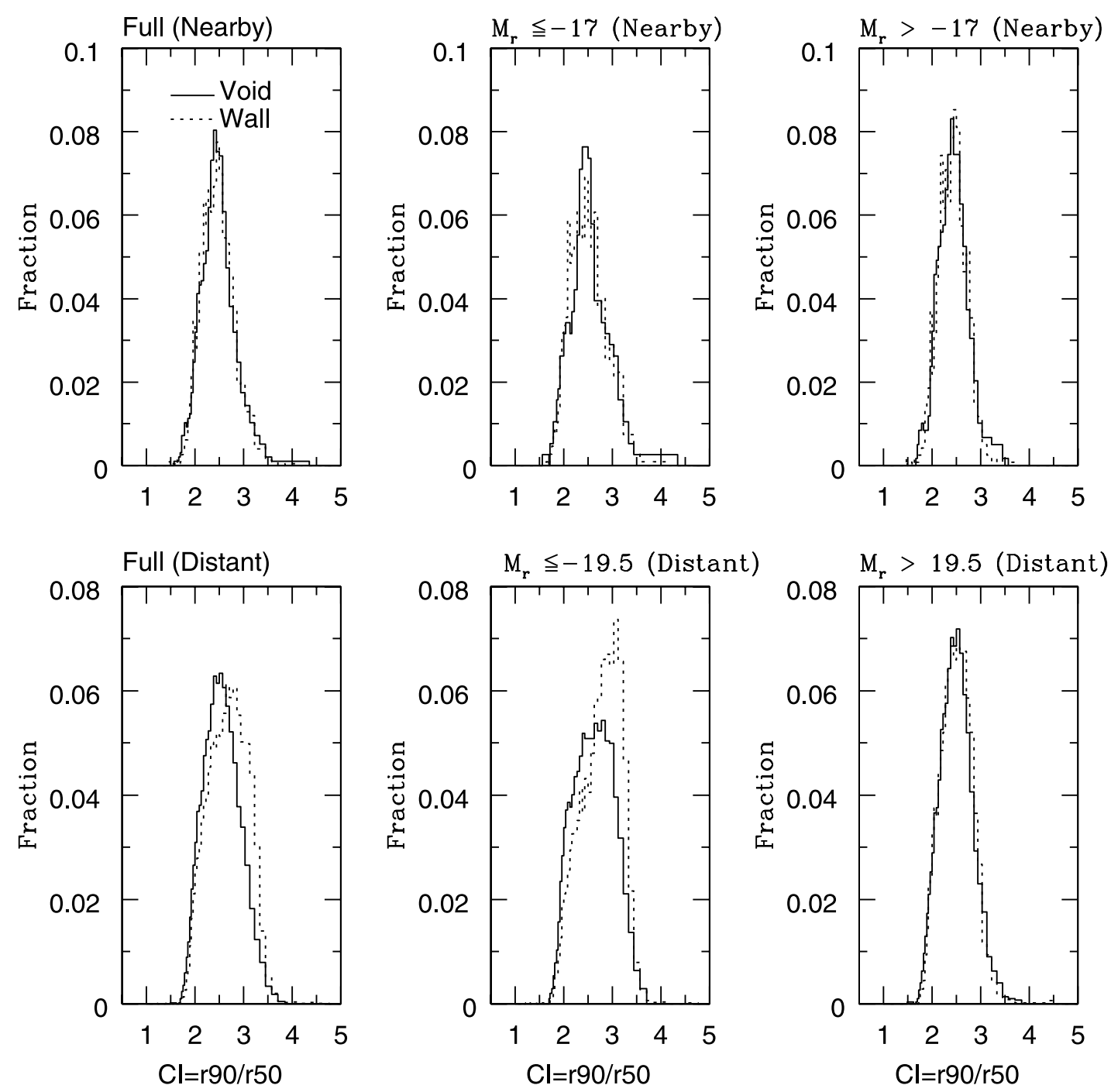

FIG. 8.-Concentration index distribution. We show the normalized fraction of void (solid lines) and wall galaxies (dotted lines) as a function of $r_{90} / r_{50}$. The top row shows the results for the nearby galaxies; the bottom row shows the results for the distant galaxies. The left, middle, and right panels are again the full, bright, and faint samples. The fraction of galaxies per 0.1 bin of concentration index is shown on the $Y$-axis. The shift in the distribution for the distant, bright $\left(M_{r} \leq-19.5\right)$ wall

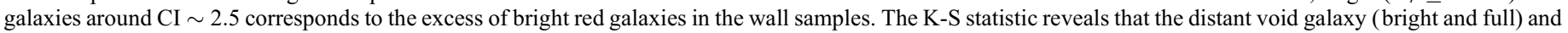

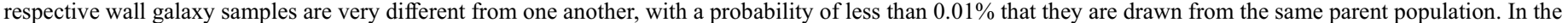
case of the nearby galaxies, the two distributions are indistinguishable. 

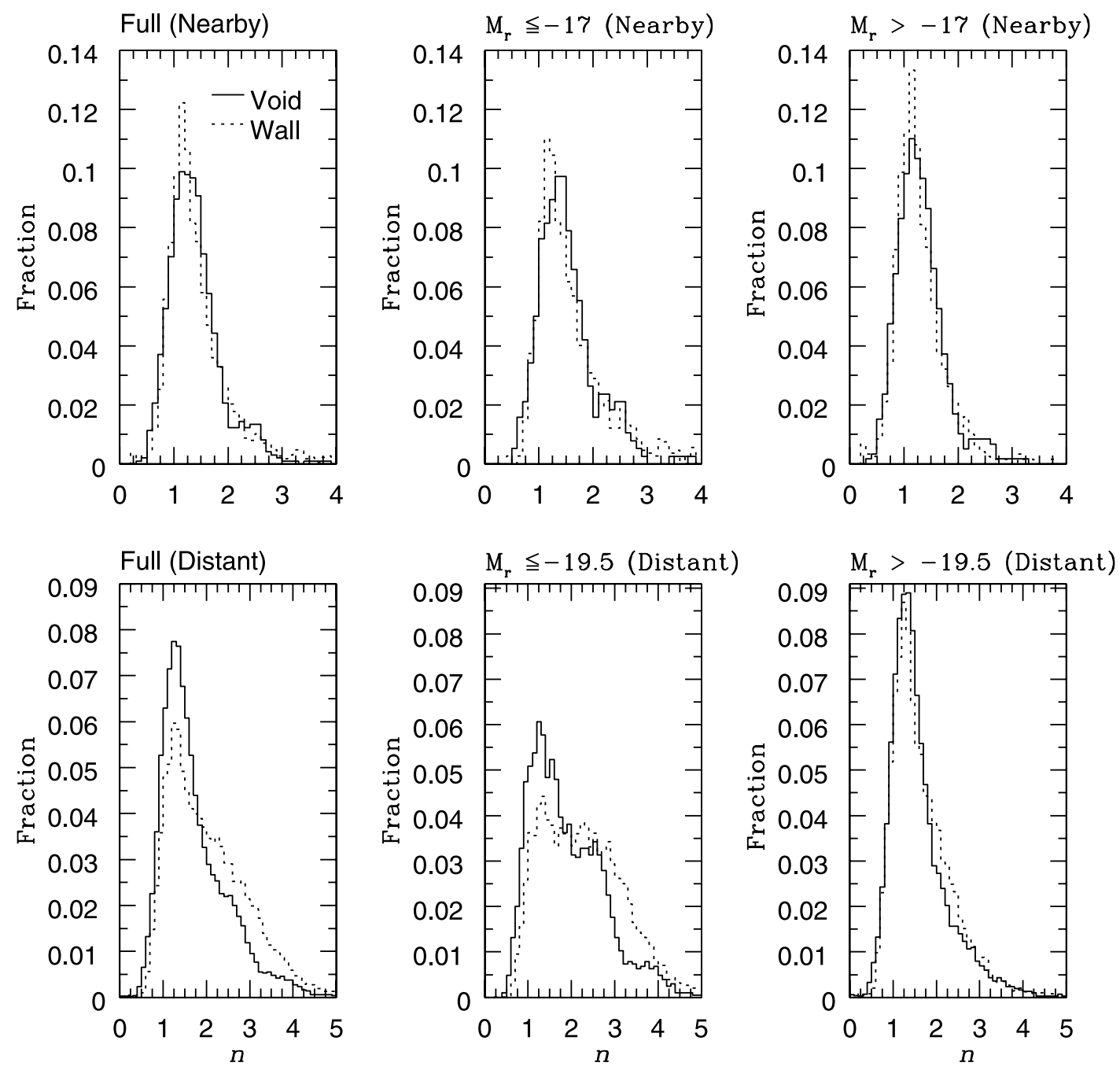

FIg. 9.-Sersic index distribution. We plot the normalized fraction of void (solid lines) and wall galaxies (dotted lines) as a function of Sersic index. The top row shows the results for the nearby galaxies; the bottom row shows the results for the distant galaxies. The left, middle, and right panels are again the full, bright, and faint samples. The fraction of galaxies per 0.1 bin of Sersic index is shown on the $Y$-axis. We cannot distinguish the nearby void galaxies from the respective nearby wall galaxy sample based on the Sersic index. However, for the distant samples, the K-S test assigns a probability of $P<10^{-4}$ that the void galaxies are drawn from the same parent population as the respective wall galaxies.

later type than wall galaxies. We include the more nearby, perhaps slightly diluted, void galaxy sample in our full analysis because it allows us to probe a larger range of absolute magnitude. In fact, we find consistency of results in the nearby and distant samples over the range of absolute magnitude where these samples overlap. The statistical significance is comparable perhaps because the nearby samples are relatively smaller, albeit purer. We expect that the statistical significance of these comparisons will rise in future, more complete samples from the SDSS.

One might ask if the observed differences in color are simply the result of the well-known morphology density relation, extrapolated down to lower densities: blue spiral galaxies are found in low-density environments, while red elliptical galaxies are found in clusters. This explanation seems unlikely in the nearby samples, where the SBPs of void and wall galaxies are quite similar. Thus, in the nearby samples, the difference in color is not clearly linked to morphology. In the distant samples, however, we see a morphological difference between the void and wall sample; there are more elliptical-type galaxies in the wall sample. To test if the difference in color is caused simply by the paucity of elliptical galaxies in voids, we divide the distant sample by Sersic index. Blanton et al. (2003b) use $n<1.5$ to represent exponential disks and $n>3$ for the de Vaucouleurs profiles, whereas Vennik et al. (1996) use $n \leq 1.5$ for exponential law fits and $n \geq 1.6$ for early-type galaxy profile fitting. We examine the color distributions of void and wall galaxies with a Sersic index less than 1.8 and greater than 1.8 to approximately split the sample into spiral and elliptical galaxies. We find that the void galaxies with both $n<1.8$ and $n>1.8$ are bluer than the wall galaxies. In $u-r$ and $g-r$ and for both $n<1.8$ and $n>1.8$ the void galaxies are at least $3 \sigma_{\mu}$ bluer than the wall galaxies, and for the $n<1.8, g-r$ case the difference rises to $7 \sigma_{\mu}$. Again, the samples are divided into bright and faint subsamples as well as by Sersic index. The void galaxies are always bluer than the wall galaxies, although the significance of the K-S test is reduced because of the smaller number of galaxies.

Thus, void galaxies are bluer than wall galaxies even when compared at similar SBPs and luminosities. They are also fainter and have SBPs that more closely resemble spiral galaxies than elliptical galaxies. These findings are consistent with predictions of void galaxy properties from a combination of semianalytic modeling and $N$-body simulations of structure 
formation in CDM models (Benson et al. 2003). One of the reasons that void galaxies are bluer than galaxies in richer environments may be that star formation is an ongoing process in void galaxies. Galaxies in clusters and groups have their supply of fresh gas cut off. Therefore, star formation is suppressed in the wall galaxies.

To illustrate the range of luminosities probed by this study, we consider which members of the LG could have been included in our samples at the distances probed by the SDSS volume. Not only the brightest members of the LG, but also LG members such as M31 and M33 can be detected in the distant sample, and fainter $\left(M_{r} \gtrsim-16.5\right)$ members of the LG, such as the Large Magellanic Cloud and Small Magellanic Cloud, would be included in the nearby sample.

In the nearby sample we can detect faint dwarf elliptical (dE) galaxies, which is to be expected given that about $80 \%$ of the known galaxies in the LG are dwarfs (Sung et al. 1998; Staveley-Smith et al. 1992). It is well known that while dE galaxies have exponential SBPs (Sandage \& Bingelli 1984; Bingelli et al. 1987; Caldwell \& Bothun 1987), they exhibit color gradients that redden outward (Jerjen et al. 2000; Vader et al. 1988; Bremnes et al. 1998) and have a uniform color distribution (James 1994; Sung et al. 1998). On the basis of their color and other properties, a fraction of the void galaxies resemble a population of $\mathrm{dE}$ galaxies, which have a mean $g-r=0.51$ (Kniazev et al. 2003). Typical dE galaxies have Sersic indices $n \sim 1.0$ and $M_{B} \gtrsim-17$, consistent with our sample of nearby void galaxies (see Table 1).

\section{CONCLUSIONS}

Using a nearest neighbor analysis, we identify void galaxies in the SDSS. For the first time we have a sample of $\sim 10^{3}$ void galaxies. These void galaxies span a wide range of absolute magnitudes, $-13.5>M_{r}>-22.5$, which are found out to distances of $260 h^{-1} \mathrm{Mpc}$ and in regions of the universe that have density contrast $\delta \rho / \rho<-0.6$.

In previous studies of void galaxy properties it was suggested (Vennik et al. 1996; Pustilnik et al. 2002; Popescu et al. 1997) that void galaxies could be distinguished from nonvoid galaxies based on their color, and a hint of them being bluer was observed (Grogin \& Geller 1999, 2000) from a small sample of void galaxies. In this paper we present a definitive result with a sample of 1204 void galaxies for which the colors, concentration, and Sersic indices are compared against wall galaxies.

Void galaxies are bluer than wall galaxies of the same intrinsic brightness and redshift distribution down to $M_{r}=$ -13.5 . We demonstrate that the difference in colors is not explained by the morphology-density relation. Nearby void and wall galaxies have very similar SBPs, and still the void and wall galaxies have different colors. In the distant sample the voids and wall galaxies have different SBPs. However, when we divide the populations further by Sersic index, the void galaxies are still bluer. To test that the differences in color are not due to the choice of absolute magnitude range, we compare the colors within narrow bins of absolute magnitude. This reveals that void galaxies are genuinely blue and that the differences between the colors are not dominated by extreme objects in the tails of the void and wall galaxy distributions.

Analysis of SBPs indicates that void galaxies are of later type than wall galaxies. Comparison of the Sersic indices between the distant void and wall galaxy samples including subsamples within a narrow range of luminosities shows that it is very unlikely $\left(P<10^{-4}\right)$ that the two samples are drawn from the same parent population. However, based on the concentration index, it is only the bright distant void and wall galaxy samples that differ significantly.

Our results are in agreement with predictions from semianalytic models of structure formation that predict that void galaxies should be bluer, fainter, and have larger specific star formation rates (Benson et al. 2003). The differences in color are probably best explained in terms of star formation. Void galaxies are probably still undergoing star formation, whereas wall galaxies have their supply of gas strangled as they fall into clusters and groups.

In a separate paper (Rojas et al. 2004) we discuss analysis of the spectroscopic properties $(\mathrm{H} \alpha,[\mathrm{O}$ II] equivalent widths, and specific star formation rates) of our void galaxies. Work in progress reveals that the specific star formation rate of our void galaxies is considerably higher, consistent with our current findings and predictions.

Funding for the creation and distribution of the SDSS Archive has been provided by the Alfred P. Sloan Foundation, the Participating Institutions, the National Aeronautics and Space Administration, the National Science Foundation, the US Department of Energy, the Japanese Monbukagakusho, and the Max Planck Society. The SDSS ${ }^{4}$ is managed by the Astrophysical Research Consortium for the Participating Institutions. The Participating Institutions are the University of Chicago, Fermilab, the Institute for Advanced Study, the Japan Participation Group, the Johns Hopkins University, Los Alamos National Laboratory, the Max-Planck-Institute for Astronomy, the Max-Planck-Institute for Astrophysics, New Mexico State University, University of Pittsburgh, Princeton University, the United States Naval Observatory, and the University of Washington. M. S. V. acknowledges support from NSF grant AST 0071201 and a grant from the John Templeton Foundation. We acknowledge David Goldberg for useful conversations. We thank the referee for valuable comments.

${ }^{4}$ The SDSS Web site is http://www.sdss.org.

\section{REFERENCES}

Abazajian, K., et al. 2003, AJ, 126, 2081

Alonso, M. V., da Costa, L., Latham, D., Pellegrini, P. S., \& Milone, A. E. 1994, AJ, 108, 1987

Babul, A., \& Postman, M. 1990, ApJ, 359, 280

Baldry, I. K., Glazebrook, K., Brinkmann, J., Ivezic, Z., Lupton, R. H., Nichol, R. C., \& Szalay, A. S. 2004, ApJ, 600, 681

Benson, A. J., Hoyle, F., Torres, F., \& Vogeley, M. S. 2003, MNRAS, 340, 160

Bingelli, B. 1989, in Large-Scale Structure and Motions in the Universe, ed. M. Mezzetti (Dordrecht: Kluwer), 47

Bingelli, B., Tammann, G. A., \& Sandage, A. 1987, AJ, 94, 251

Blanton, M. R., Lin, H., Lupton, R. H., Maley, F. M., Young, N., Zehavi, I., \& Loveday, J. 2003a, AJ, 125, 2276
Blanton, M. R., et al. 2001, AJ, 121, 2358

2003b, ApJ, 594, 186

2003 c, AJ, 125,2348

Bremnes, T., Bingelli, B., \& Prugniel, P. 1998, A\&AS, 129, 313

Caldwell, N., \& Bothun, G. D. 1987, AJ, 94, 1126

da Costa, L. N., et al. 1998, AJ, 116, 1

Dekel, A., \& Silk, J. 1986, ApJ, 303, 39

Dressler, A. 1980, ApJ, 236, 351

Efstathiou, G., Ellis, R. S., \& Peterson, B. S. 1988, MNRAS, 232, 431

Eisenstein, D. J., et al. 2001, AJ, 122, 2267

El-Ad, H., \& Piran, T. 1997, ApJ, 491, 421

El-Ad, H., Piran, T., \& da Costa, L. N. 1996, ApJ, 462, L13 
El-Ad, H., Piran, T., \& da Costa, L. N. 1997, MNRAS, 287, 790

Evrard, A. E., et al., 2002, ApJ, 573, 7

Falco, E. E., et al. 1999, PASP, 111, 438

Frenk, C. S., et al. 2000, preprint (astro-ph/0007362)

Fukugita, M., Ichikawa, T., Gunn, J. E., Doi, M., Shimasaku, K., \& Schneider, D. P. 1996, AJ, 111, 1748

Geller, M. J., \& Huchra, J. P. 1989, Science, 246, 897

Grogin, N. A., \& Geller, M. J. 1999, AJ, 118, 2561 2000, AJ, 119, 32

Gunn, J. E., et al. 1998, AJ, 116, 3040

Hoffman, Y., Silk, J., \& Wyse, R. F. G. 1992, ApJ, 388, L13

Hogg, D. W., Finkbeiner, D. P., Schlegel, D. J., \& Gunn, J. E. 2001, AJ, 122,2129

Hogg, D. W., et al. 2003, ApJ, 585, L5

Hoyle, F., \& Vogeley, M. S. 2002, ApJ, 566, 641

Huchra, J. P., Geller, M. J., \& Corwin, H. 1995, ApJS, 99, 391

Huchra, J. P., Geller, M. J., de Lapparent, V., \& Corwin, H. 1990, ApJS, 72,433

Huchra, J. P., Vogeley, M. S., \& Geller, M. J. 1999, ApJS, 121, 287

James, D. A. 1994, MNRAS, 269, 176

Jerjen, H., Binggeli, B., \& Freeman, K. C. 2000, AJ, 119, 593

Karachentsev, I. D., \& Makarov, D. A. 1996, AJ, 111, 794

Kirshner, R. P., Oemler, A., Jr., Schechter, P. L., \& Shectman, S. A. 1981, ApJ, 248, L57

Kniazev, A. Y., Grebel, E. K., Pustilnik, S. A., \& Kniazeva, T. F. 2003, Astron. Nachr., 324, 81

Kuhn, B., Hopp, U., \& Elaässer, H. 1997, A\&A, 318, 405

Lupton, R. H., Gunn, J. E., Ivezić, Ž., Knapp, G. R., Kent, S., \& Yasuda, N. 2001, in ASP Conf. Ser. 238, Astronomical Data Analysis Software and Systems X, ed. F. R. Harnden, Jr., F. A. Primini, \& H. E. Payne (San Francisco: ASP), 269

Lupton, R. H., Gunn, J. E., \& Szalay, A. S. 1999, AJ, 118, 1406

McLin, K. M., Stocke, J. T., Weymann, R. J., Penton, S. V., \& Shull, J. M. 2002, ApJ, 574, L115

Mo, H. J., McGaugh, S. S., \& Bothun, G. D. 1994, MNRAS, 267, 129

Müller, V., Arbabi-Bidgoli, S., Einasto, J., \& Tucker, D. 2000, MNRAS, 318,280

Park, C., Vogeley, S. M., Geller, M. J., \& Huchra, J. P. 1994, ApJ, 431, 569
Peebles, P. J. E. 2001, ApJ, 557, 495

Pellegrini, P. S., da Costa, L. N., \& de Carvalho, R. R. 1989, ApJ, 339, 595

Pence, W. 1976, ApJ, 203, 39

Pier, J. R., Munn, J. A., Hindsley, R. B., Hennessy, G. S., Kent, S. M., Lupton, R. H., \& Ivezić, Ž. 2003, AJ, 125, 1559

Plionis, M., \& Basilakos, S. 2002, MNRAS, 330, 399

Popescu, C., Hopp, U., \& Elaässer, H. 1997, A\&A, 325, 881

Postman, M., \& Geller, M. J. 1984, ApJ, 281, 95

Pustilnik, S. A., Martin, J.-M., Huchtmeier, W. K., Brosch, N., Lipovetsky, V. A., \& Richter, G. M. 2002, A\&A, 389, 405

Rojas, R. R., et al. 2004, ApJ, submitted

Sandage, A., \& Binggeli, B. 1984, AJ, 89, 919

Schlegel, D. J., Finkbeiner, D. P., \& Davis, M. 1998, ApJ, 500, 525

Scranton, R., et al. 2002, ApJ, 579, 48

Sersic, J. L. 1968, Atlas de Galaxias Australes (Cordoba: Obs. Astronómico)

Shectman, S. A., et al. 1996, ApJ, 470, 172

Shimasaku, K., et al. 2001, AJ, 122, 1238

Smith, J. A., et al. 2002, AJ, 123, 2121

Staveley-Smith, L., Davies, R., \& Kinman, T. D. 1992, MNRAS, 258, 334

Stoughton, C., et al. 2002, AJ, 123, 485

Strateva, I., et al. 2001, AJ, 122, 1861

Strauss, M. A., et al. 2002, AJ, 124, 1810

Sung, E., Han C., Ryden, B. S., Chun, M., \& Kim, H. 1998, ApJ, 499, 140

Tegmark, M., Hamilton, A. J. S., \& Xu, Y. 2002, MNRAS, 335, 887

Thuan, T. X., Gott, J. R., III, \& Schneider, S. E. 1987, ApJ, 315, L93

Vader, J. P., Vigroux, L., Lachieze-Rey, M., \& Souviron, J. 1988, A\&A, 203, 217

Vennik, J., Hopp, U., Kovachev, B., Kuhn, B., \& Elsässer, H. 1996, A\&AS, 117,261

York, D. G., et al. 2000, AJ, 120, 1579

Zwicky, F., \& Herzog, E. 1962-1965, Catalogue of Galaxies and Clusters of Galaxies, Vol. II-IV (Pasadena: Caltech)

Zwicky, F., Herzog, E., \& Wild, P. 1961, Catalogue of Galaxies and Clusters of Galaxies, Vol. I (Pasadena: Caltech)

Zwicky, F., Karpowicz, M., \& Kowal, C. 1965, Catalogue of Galaxies and Clusters of Galaxies, Vol. V (Pasadena: Caltech)

Zwicky, F., \& Kowal, C. 1968, Catalogue of Galaxies and Clusters of Galaxies, Vol. VI (Pasadena: Caltech) 